\title{
AN EFFICIENT FOG ENABLED HEALTHCARE MAINTENANCE SYSTEM USING INTERNET OF THINGS WITH DEEP LEARNING STRATEGIES
}

\author{
G.S.Gunanidhi ${ }^{1}$, R.Krishnaveni ${ }^{2}$ \\ ${ }^{1}$ Research Scholar,Department of Computer Science, Hindustan Institute of Technology and Science \\ ${ }^{2}$ Professor, Department of Computer Science, Hindustan Institute of Technology and Science
}

\begin{abstract}
Internet of Things (IoT) is the ruling term nowa-days, in which it attracts several smart gadgets and application due to its robust nature and support. In healthcare industry several new technologies are required to improve the stability and provide transparent services to clients. The integration of healthcare maintenance system with respect to Internet of Things support leads to a drastic change in healthcare field as well as this provision provides huge advantages to users. This paper is intended to provide an intense healthcare maintenance scheme by using latest technologies such as Deep Learning, Internet of Things, Fog Computing and Artificial Intelligence. All these innovations are associated together to build a new deep learning strategy called Intense Health Analyzing Scheme (IHAS), in which this proposed approach provides all provisions to clients such as Doctors and Patients with respect to monitor the patient details from anywhere at anytime without any range boundaries. The Fog Computing is an innovative domain, in which it provides ability to the server to operate based on hurdle free processing logic. Artificial Intelligence logic is used to manipulate the health data based on previously trained health records, so that the predictions are more fine compare to the classical healthcare schemes. In traditional schemes it is difficult to raise an alert based on the emergency situation predictions, but in the proposed deep learning strategy assists the proposed approach to send an alert instantly if any emergency cases occurred on patient end. Generally the Fog Servers are used to reduce the occupancy of the storage server and provide reliable storage abilities to server, but in this proposed approach, the fog server is utilized for priority wise data handling nature and stores the health records accordingly. In this nature, the fog servers are handled and provide high efficient results to the clients in an innovative way. With the help of deep learning procedures, the health records are clearly prioritized and maintained into the server end for monitoring. For all this paper introduced a new logic of healthcare maintenance scheme IHAS to provide efficient support to patients as well as doctors in clear manner.
\end{abstract}

Keyword: Internet of Things, IoT, Fog Server, Deep Learning, Artificial Intelligence, Healthcare System, Intense Health Analyzing Scheme, IHAS

\section{Introduction}

Healthcare is one of the major domain now-a-days, each and every individual requires more attention on it as well as the improvement level of healthcare oriented systems are also drastically changed. The advancement of latest technology devices provides more features to rise up the classical healthcare systems into a modernized one. The consideration of privacy in healthcare applications are must, so that nobody can access the system without proper credentials. These are all the constraints to deal with healthcare applications in more depth. Internet of Things generally termed as a refined form of network, in which it accumulates the local sensor enabled data and pushed that accumulated data into the remote server with proper access mechanisms. The remote server is a cloud server environment, in which it can accumulate huge amount of health records in one place for future accessing and references. But the concept of such cloud services is diplomatic in terms of two constraints such as storage and accessing expensiveness as well as the privacy issues. The health data collected from the local hospital environments from the smart sensor units and pass that to the remote server via any service provider is a general case, but there is a possibility of attackers or intruders to harm the data and there is a lot of chances to modify the health records of the patients. This will cause a huge destruction in medication environments. The next term of storage cost or expensiveness creates an issue like the continuous health records requires a huge server unit to maintain all the health data. So that the requirement is high, in which it leads a huge cost to manage such server in remote end. To provide a solution for such quoted hurdles, a new category of healthcare maintenance scheme is required to resolve the issues and provides a great support to medication field. In this paper, a BigData based Cloud Server management scheme is introduced, in which the data size may be anything but the server end is handled by the BigData server, so that there is no chance to data overhead and data loss. 
All the accumulated data is properly maintained into the server end without any failure chances of the proposed approach. The resulting unit clearly proves this by means of graphical visualizations. The concept of deep learning principles is introduced to manage the healthcare predictions, in which the patient health status is easily predicted with the help of such deep learning principles. The concept of Deep Learning is basically derived from Artificial Intelligence, in which it regrets the operational process of the human in terms of decision making and choice estimations in clear manner. The same category of deep learning principles is applied to the proposed approach to provide a multi-level processing ability to the system. The training principles of the proposed approach is unique, because the coming health record is considered for the further testing process, so that the dynamic training model creation principle is happened over this system to provide maximum level of accuracy to the proposed approach. Fog processing unit is utilized in smart gadgets, for example, smart-watch, in which it doesn't just show timeperiod, however shows the means taken by the individual including how far strolled and the calories consumed. The gadget can gauge the heart beat level and make the individual hard or delicate rest. The smart-mobile phones these days outfit themselves with in-constructed sensors such as heart rate estimation sensors, gyrometer, accelerometer fused in advanced mobile phones [1][2]. Fog computing is an allinclusive element of distributed computing, where both processing stages have comparable admissions which are invaluable to fog registering with utilization of inertness decrease in cloud storage units. The fog nature of distributed computing environments makes a total bundle where it comforts the clients, yet additionally has inconveniences. One of the benefits of Internet of Things is rapid handling and computing of enormous information, produced and oversaw productively in different applications [3]. Keen consideration frameworks are needed, specifically, in quick changing speed of human existence. At the point when the fog system processing and IoT are introduced in machines pertinent to clinical industry applications, the productivity of the tasks can be improved in such medical services frameworks [4].

The new frameworks are far superior to the past or customary frameworks as more dependable and effective gadgets are utilized with information correspondence innovations that have security compatibilities. The medical care costs are diminished [8] by introducing the fog system processing and Internet of Things. With speed up the processing and preparing nature of the fog systems, the nature of healthcare system is improved. The improvement is seen on a more prominent greatness with wellbeing observing done, in light of Internet of Things framework with fog processing [9]. The principle preferred position of utilizing Internet of Things in clinical medical services is to diminish boundaries in checking significant health boundaries and lessening pointless healthcare costs [5][10]. The Smart wearable gadgets are for the most part being used in medical care field, through which it is the least demanding and an agreeable method to accumulate the information, screen health and impart general their experts occasionally. The smart gadgets help Internet of Things to change the essence of medicine through their capacities and systems. The methods and capacities are fundamental segments of Internet of Things for information era, social occasion and the board. Furthermore, the gadgets dissect information, utilize a bunch of decides and procedures that required in the investigation. Health trackers show up in tremendous amounts which work over the biggest measure of datasets and the analysis of datasets are performed to know the size of health-related approaches of patients, being the information are from numerous areas and applications. The appliance of Global Positioning System (GPS) and the associated sensors are appended as smart gadgets, similar to inhalers are connected to trackers for several category of patients (Ex. Asthma Patients). Detecting the difference in area, the climate around the patient, observing the drug timings are instances of exercises of the gadgets as well as the smart gadgets and sensors alert with different realities that outcome huge measure of information assortment from patients through tracks and notices. All these related details will be explained clearly over the further methodologies section in detail. The following figure, Fig-1 illustrates the overall architecture of the proposed healthcare systems in detail with diagrammatic representation.

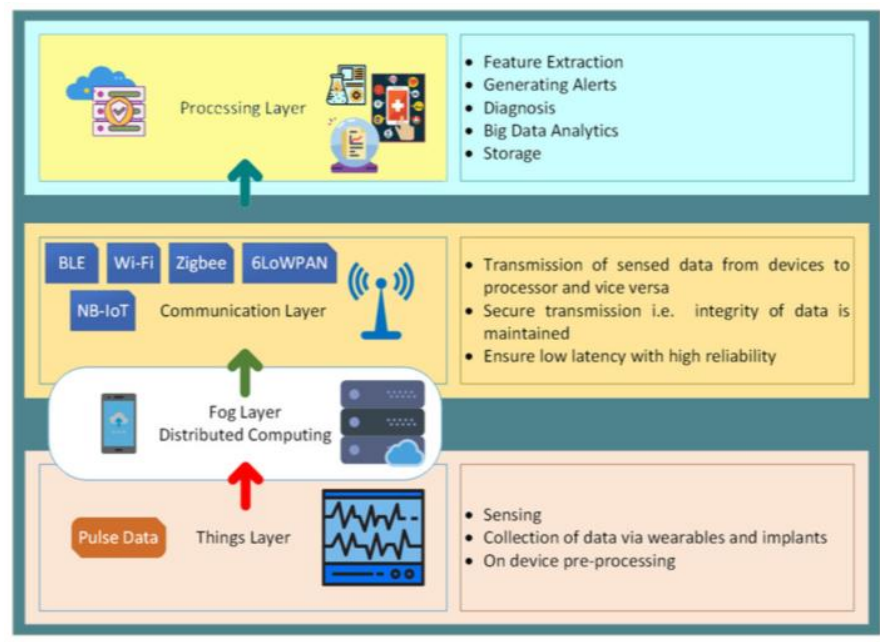

Fig.1 Proposed System Architecture

The rest of this paper describe regarding Related Study over section 2, further section of Section 3 illustrates the proposed system methodologies in detail with proper algorithm flow and the Section 4 illustrates the Result and Discussion portion of the paper and the final section, Section 5 illustrates the concept of Conclusion of the proposed paper. These all will be explained in detail over the further section summaries. 


\section{Related Study}

In the year of 2020, the authors "MuhibAnwarLambay'et al., [5]" proposed a paper related to the implementation of healthcare systems using BigData logic in association with Distributed File Management System. In this paper [5], the authors described such as: Healthcare field is an imperative element in reality where enormous volumes of information is gathered now and again. Such information accepts qualities of huge datat and it is attractive to dissect it and achieve idle connections among factors in the healthcare information. Information in medical services field is wealthy in valuable data and in any case, an extensive BigData approach is vital for mine the information and get business insight [5]. There are many use instances of BigData analysis. Nonetheless, in healthcare industry it is basic to have information driven suggestions that help all researchers. With the development of distributed file management system and computing process, BigData investigation has become a reality. Dispersed programming structures like Hadoop-and-Spark, to make reference to not many, are accessible with related Distributed File Management System to oversee BigData. Numerous analysts contributed towards creating techniques dependent on Artificial Intelligence, in which it is important for machine learning principles. Till now healthcare field is one of the wellsprings of BigData, it needs dispersed conditions for preparing. The distributed file management system principle and BigData environment is assuming great part in acknowledging enormous information analysis for medical service proposals. This paper [5] tosses light into various recommenders in medical services area that utilization BigData investigation to produce suggestions as well as it gives helpful bits of knowledge as well as talked about exploration holes that can be utilized to examine further to improve the best in class.

In the year of 2020, the authors "HudiartoSukarman'et al., [6]" proposed a paper related to healthcare framework in terms of Enterprise Architecture environment. In this paper [6], the authors described such as: medical factors are more important in case of monitoring individual health status as well as such monitoring mechanisms provide a drastic view of handling such information in digital manner. But in some countries still it is a complex task to manage such applications, the country Indonesia needs some sort of applications with respect to machine learning strategies in association with developed countries such as Singapore, Malaysia and Australia. Because of wealth reasons and the money factors these countries get a drastic development in healthcare maintenance approach and provide a constant support to their patients in an innovative way. This paper [6] concentrates more on healthcare industry with respect to the adaptation of latest technologies such as machine learning and information technology domains. The present industry competitions and the strict rules of government, these system implementations are tough in certain countries. Due to this kind of problems this paper [6] utilizes the advantage of Enterprise-Architecture Environment EA3 to resolve all such issues quoted above and provides a solution to the problems by means of funding sources and provide development opportunities and this motivation with this plan of the data framework as well as innovation procedure, interest in the field can be coordinated and the business goals that have been set can be accomplished.

In the year of 2020, the authors "SabyasachiChakraborty'et al., [7]" proposed a paper related to healthcare optimization procedures and provide a drastic support to patients in terms of customized application view and the related provisions. In this paper [7], the authors described such as: the point of this work is to enhance the medical care network for improving help conveyance by distribution and de-allocation of medical services assets according to require. The extrapolation of the requirement for environments and assets are the most testing part of this work which requires information on the number of inhabitants in different geographic districts/regions. The populace is additionally taken a gander at from the point of view old enough gathering, economy, the normal part of populace requiring medical services and furthermore remembering any set of experiences of sicknesses or such inclined zones. Conjecturing the thumb rule of the medical services specialist to tolerant proportion for example $1 / 2808$ to make work smooth and patient consideration heartier for the employees. The proposed endeavors are to help the U.S. Washington health service to revise, create and improve the employee structure and assets in existing environments.

\section{Proposed System Methodologies}

In this paper, a new logic is introduced to provide efficient healthcare mechanisms in terms of deep learning procedure called Intense Health Analyzing Scheme (IHAS). This approach integrates several latest technologies together to provide an intellectual health data processing and alert scheme. The associated technologies such as: BigData, Deep Learning, Internet of Things, Fog Computing and Artificial Intelligence provide a great support to the proposed approach. All these will be integrated together into a smart device and that can collect health related data from the patient and pass it to the remote cloud server via Internet of Things module. The data will be processed on the server end with Deep Learning procedures and maintain the processed data into the server with proper prioritization logic. In this deep learning approach of Intense Health Analyzing Scheme provides a sufficient way to manage health care details with respect to connected sensors called Heart Rate Monitoring Sensor, Gyroscope Sensor, Room Temperature and Humidity Estimation Sensor, Body Temperature Monitoring Sensor and the Global Positioning System (GPS). The data is transmit from smart gateway to Fog Layers, which Fog
ISSN (Print): 2204-0595 ISSN (Online): 2203-1731 
have some important services such as data pre-processing ,cleaning, low latency, response time, and etc. All these functions and layer principles are explained in detail over the following summary. The motto of the proposed approach IHAS is to provide the following options to the users.

- Simple and portable circuit connections.

- Cost efficient implementation logic.

- Scalable and Robust Design.

- This system has the ability of using by common people, especially children and aged people, and don't need any special training.

- Requires Less Power consumption and Compatible in size. Healthcare Scheme with Fog Interface

The fog computing interface is adapted with the proposed deep learning principle in terms of reducing the data storage over the remote cloud server and providing the secured data maintenance space on the server end. This is done by means of prioritization logic. The servers are deviated into two forms, such as internal and external. In which the internal servers are utilized once the health related details has low priority and the high priority health data are stored into the remote IoT cloud servers.

This kind of processing logic totally eliminates the data loss and improves the processing time in good manner with high success ratio. The prioritization prediction logics are attained by means of Deep Learning procedure, in which it classifies the health data based on the threshold level. The processed health data crosses the threshold level that will be considered as a high priority data and the remaining all are treated as a general data. The general or regular periodical health related details are maintained into the local server maintained into the hospital end and the high priority health data such as critical health condition of patients such heart rate abnormal and so on will be stored into the remote cloud server. This kind of fog nature improves the processing power of the system and provides an efficient healthcare monitoring approach in nature. The following figure, Fig-2 illustrates the fog computing working model in graphical manner.

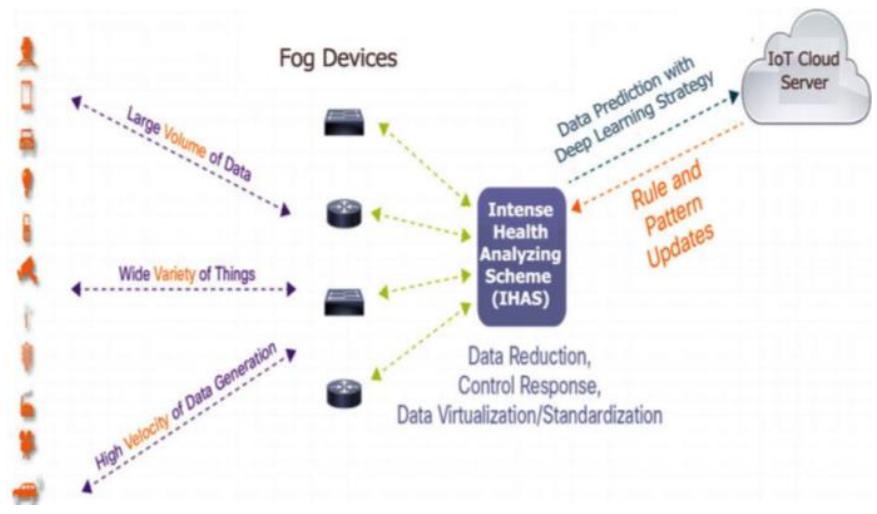

Fig.2 Fog Server Process Working Model

Intense Health Analyzing Scheme (IHAS)
This paper introduces a new deep learning principle called Intense Health Analyzing Scheme (IHAS), in which it integrates several applications to provide a huge support to healthcare analysis. The concept of BigData is utilized over the approach to provide efficient data handling scheme, because the data coming from the hospital environment is huge and it periodically come from the smart device without any predictable ratio. So, that a huge support is required to manage such data in the server end. The concept of BigData is associated with the proposed approach to provide such facility to handle the server end in intense manner. The following are the parameters used to analyze the proposed approach of IHAS healthcare performance summary, Processing Time, Availability, Reliability, Response Time and Storage ability. The following table, Table-1 illustrates the proposed approach processing ability in terms of quoted parameters in detail.

Table-1 Performance Estimation Parameters

\begin{tabular}{|l|l|}
\hline Parameters & Specification \\
\hline Processing Time & $\begin{array}{l}0.2 \mathrm{~ms} \text { for } 10 \text { sequential health } \\
\text { records. }\end{array}$ \\
\hline Availability & $\begin{array}{l}\text { 24/7 with same specification. } \\
\text { real time evaluations. }\end{array}$ \\
\hline Reliability & $\begin{array}{l}\text { Based on the processing time it varies } \\
\text { but in the proposed approach, a fog } \\
\text { nature reduces the time complexity. }\end{array}$ \\
\hline Storage Ability & $\begin{array}{l}\text { The fog and BigData provides a huge } \\
\text { support to maintain n number of } \\
\text { health records without any hurdle. }\end{array}$ \\
\hline $\begin{array}{l}\text { Power } \\
\text { Requirements }\end{array}$ & $\begin{array}{l}\text { The overall Smart Device size is upto } \\
5 \text { to } 7 \text { inches maximum and the power } \\
\text { consumption of such device is not } \\
\text { more than 5v DC. So that a battery } \\
\text { power is enough to run the system in a } \\
\text { whole day without any hurdle. }\end{array}$ \\
\hline The following
\end{tabular}

The following algorithm illustrates the step by step flow levels of proposed approach called Intense Health Analyzing Scheme (IHAS) in effective manner with proper Pseudocode specifications.

\footnotetext{
Algorithm: Intense Health Analyzing Scheme

Input: Health Record Accumulated from Smart Device (Heartbeat (Hb), Blood Pressure (Bp), Temperature (Tmp), Humidity (Hm),
} 
Body Temperature (BT), Position (Pos).

Output: Check and return the priority level with proper predictions.

Step-1: Collect the health records from the hospital environment, in which it is accumulated from smart device sensor values.

Step-2: Call the required Deep Learning libraries to process the health records with respect to tensorflow open source library.

Step-3: Define the required libraries to perform health record processing with respect to keras model.

Step-4: Define the respective variable for processing the health assisted values.

\section{Pseudocode:}

Import tensorflow from tensor.

Import keras from tensor tensorflow

from keras import Data_Generator;

heartrate $=\mathrm{Hr} ;$ BloodPressure $=\mathrm{Pr} ;$ RoomTemperature $=\mathrm{Tmp}$;

BodyTemperature=BT; Humidity $=\mathrm{Hm}$; Position=Pos;

Step-4: Check the accumulated value ranges are within the defined threshold level.

Step-5: If all the Sensor level meets the threshold value ranges then the priority is set to be LOW or else it is set to be HIGH.

Step-6: If the priority level is HIGH, then check for duplication of content with respect to particular time intervals.

Step-7: If the priority level is indicated as duplicate, then the values are not maintained into the server or else the corresponding health records are properly maintained into the server.

Step-8: Return the priority value with prediction result.

Pseudocode:

$$
\text { return priority(prediction); }
$$

Step-9: Maintain the data into the remote IoT server with respective priority level.

\section{Results and Discussions}

In this description, the experimental proof of the proposed approach Intense Health Analyzing Scheme (IHAS) is discussed with proper graphical outcomes. This system of healthcare maintenance is implemented on multi-level fog environment generated server platform, in which it generates many small servers for local health record maintenance and the low priority regularized data are maintained into this local server and the periodical backup procedures are also handled by the respective system administrator for every one week of interval. The fog maintains the top end prioritization server on the remote IoT cloud server end, in which the high priority health records are stored into that server with proper alert principles. The duplicate data is identified over the server processing by means of classification principles generated by the deep learning procedures. The remote end monitoring and verification logics are generated with the help of Android Studio and the Python server end scripting, so that the user can easily monitor the health condition of the respective patients without any hurdle. The data collected in the server end can easily be monitored by the respective care-taker and the associated doctor, so that the manual error probabilities are highly reduced and the prediction accuracy level of the proposed approach is around $95.9 \%$ with less error ratio. The purpose of using such open source tools are cost effective and provides the response time as simpler than the other scripting tools, this proposed approach of IHAS is implemented by using Python Scripting language and attains the best accuracy range as well as the prediction logic. The following, figure, Fig-3 provides a detailed graphical output for the estimation of health records acquiring accuracy ratio and the corresponding error rate while data accumulation, in which the $\mathrm{x}$-axis indicates the data collection range and the y-axis indicates the accuracy levels and the bit error rate.

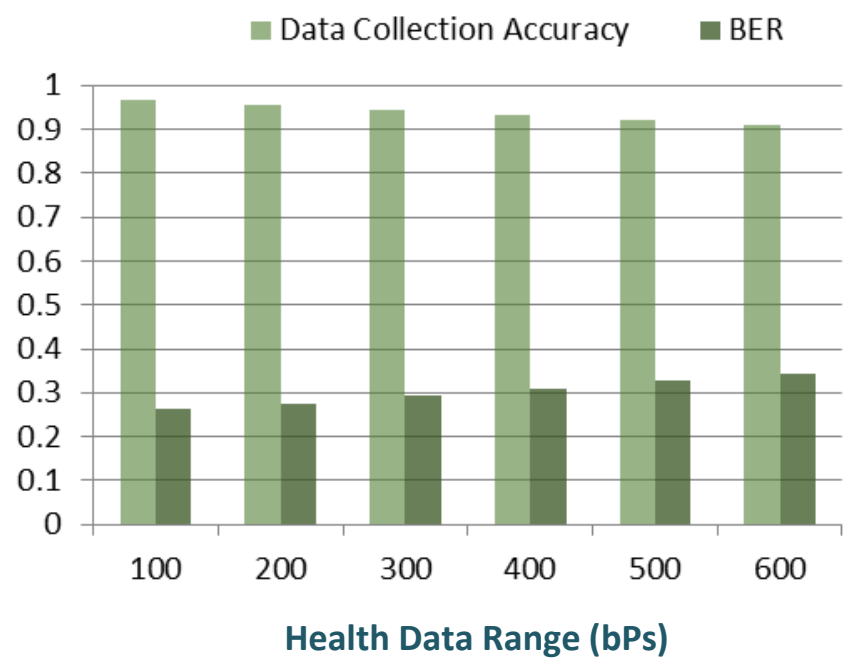

Fig.3 Data Collection Accuracy Level with respect to BER

The following figure, Fig-4 illustrates the data transmission range from the Internet of Things (IoT) module and the data reception in the Fog Server ranges in terms of $2.65 \mathrm{GHz}$ internet transmission frequency range. These values are tested in real time hospital environment for more than 2 months of timeline in continuous manner. In which the $\mathrm{x}$-axis indicates the data transmission range, that is generated based on the Smart Device sensor units and the $y$-axis indicates the data reception range on the fog cloud server. 


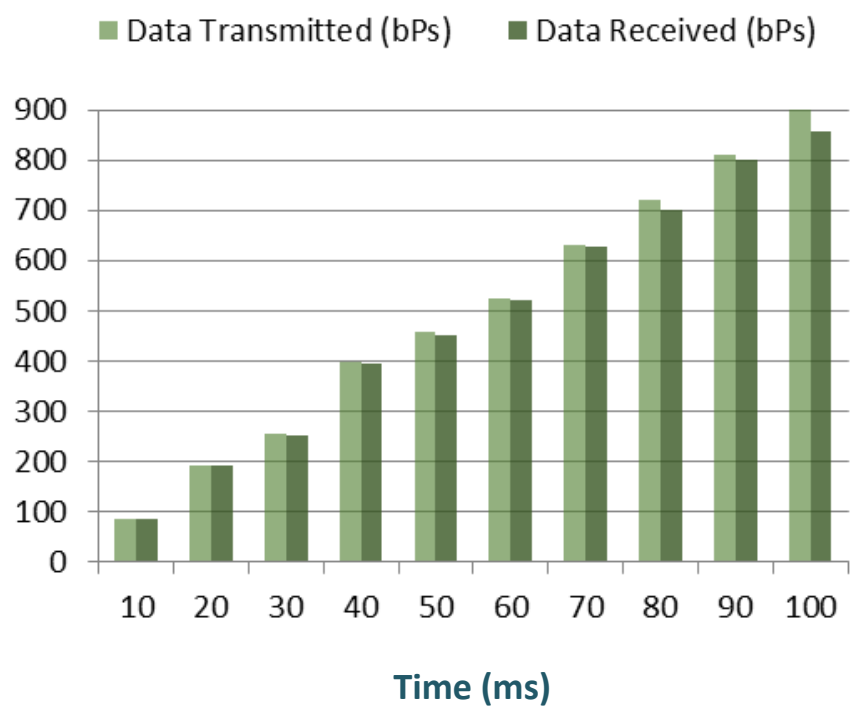

Fig.4 Data Transmission and Reception Level Estimations The following figure, Fig.5 demonstrates the proposed deep learning strategy Intense Health Analyzing Scheme (IHAS) data maintenance ratio with respect to priority ranges, in which it shows the number of high priority data predicted, number of low priority data predicted with respect to the received health record.

\section{Data Range (bPs) घ High Priority $\quad$ Low Priority}

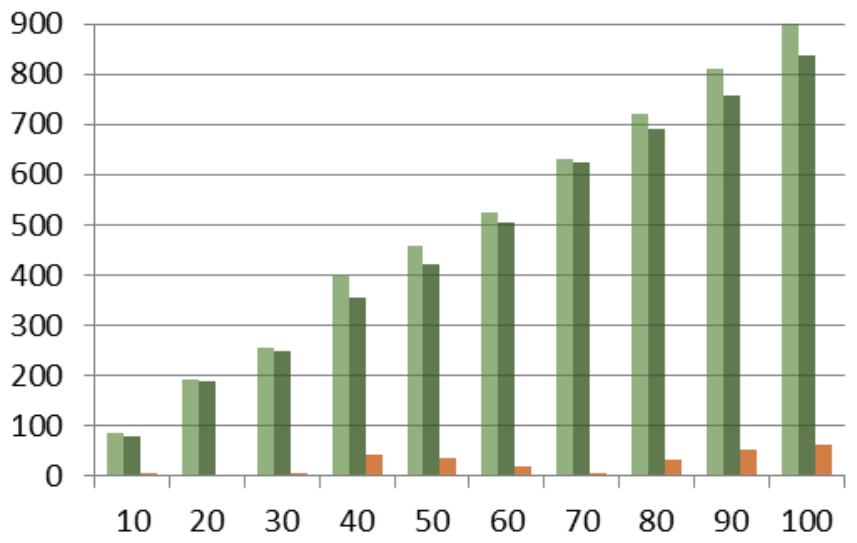

\section{Fig.5 Health Records Accumulation Ratio vs. Low and High Priority}

\section{Conclusion}

In this paper, a clear analysis of healthcare monitoring system is defined with proper parametric constraints based on Deep Learning principles. This paper introduces a new deep learning strategy called Intense Health Analyzing Scheme (IHAS), in which it integrates several latest innovations into it such as BigData, IoT, Fog Processing and AI. These all features are associated to provide an efficient healthcare analysis process in an efficient manner. This system provides a provision to care takers to view the respective patient health related details in remote manner without any hurdles and range specifications. The proposed approach of IHAS provides an accuracy range of $95.9 \%$ and the bit error ratio is too low compare to the classical machine learning approaches and the resulting section proves the efficiency with graphical proof in figure, Fig-3 and Fig-4. This approach assures the proper privacy maintenance scheme with respect to deep learning principles, so that the respective care taker or doctor need to access the system, in which it turns require the proper credentials to access the features further, otherwise the entire system is inaccessible to the user. These provisions makes the system perfect with respect to healthcare associations and the medication industry can get more benefits based on the appliance of such application.

\section{References}

[1]. Inas S. Khayal, "Dynamic Modeling of Complex Healthcare Systems Using Big Data to Describe and Visualize Healthcare Utilization", IEEE International Systems Conference (SysCon), 2020.

[2]. Direk Sueaseenak, Ploypilin Thongnawakun, et al., "Development of Personal Heart Healthcare System to Promote a Wellness of Thai People", International Electrical Engineering Congress (iEECON), 2020.

[3]. V. R. Brus, L. I. Voronova and V. I. Voronov, "Neural Network Classification of Cardiac Activity Based on Cardiogram Data for Driver Support System", Systems of Signals Generating and Processing in the Field of on Board Communications, 2020.

[4]. Mutaz Zalloum and Hosam Alamleh, "Privacy Preserving Architecture for Healthcare Information Systems", IEEE International Conference on Communication, Networks and Satellite, 2020.

[5]. Muhib Anwar Lambay and S. Pakkir Mohideen, "Big Data Analytics for Healthcare Recommendation Systems", International Conference on System, Computation, Automation and Networking (ICSCAN), 2020.

[6]. Hudiarto Sukarman, Fathan Yunicha Rizkiyana, Apriyanto and Muhammad Farhan Al Farizi, "The Design of Information System and Technology Strategy for Improving Performance of Healthcare Service With EA3 Framework: (Case Study: Summit)", International Conference on Information Management and Technology (ICIMTech), 2020.

[7]. Aseer Ahmad Ansari, Sabyasachi Chakraborty, Satyabrata Aich, Beom Su Kim and Hee-Cheol Kim, "Optimization of Healthcare Network for improved service delivery", International Conference on Advanced Communication Technology (ICACT), 2020.

[8]. Poonam Shelar and Uttara Gogate, "Performance Evaluati 
on of FMAC Protocol in Healthcare Monitoring System based on Wireless Sensor Network", International Conference on Smart Innovations in Design, Environment, Management, Planning and Computing, 2020.

[9]. Mahdi Nikooghadam and Haleh Amintoosi, "An Improved Secure Authentication and Key Agreement Scheme for Healthcare Applications", International Computer Conference, Computer Society of Iran, 2020.

[10]. Radu Papara, Ramona Galatus and Loredana Buzura, "Virtual Reality as Cost Effective Tool for Distance Healthcare", International Conference on Transparent Optical Networks, 2020. 